\title{
Spatial Information in Autonomous Modal Parameter Estimation
}

\author{
Randall J. Allemang and Allyn W. Phillips \\ Department of Mechanical and Materials Engineering, College of Engineering and Applied Science, \\ University of Cincinnati, Cincinnati, $\mathrm{OH}$ 45221-0072, USA
}

Correspondence should be addressed to Randall J. Allemang; randall.allemang@uc.edu

Received 13 July 2013; Accepted 20 January 2014; Published 17 July 2014

Academic Editor: Nuno Maia

Copyright (C) 2014 R. J. Allemang and A. W. Phillips. This is an open access article distributed under the Creative Commons Attribution License, which permits unrestricted use, distribution, and reproduction in any medium, provided the original work is properly cited.

\begin{abstract}
Recent work with autonomous modal parameter estimation has shown great promise in the quality of the modal parameter estimation results when compared to results from traditional methods by experienced users. While autonomous modal parameter estimation means slightly different things to different researchers and practitioners, for the purpose of this discussion, autonomous will require an automated procedure which sorts and processes a large number of possible modal parameter solutions to yield one consistent estimate with no user interaction after initial thresholds are chosen. In the work discussed, this final, consistent set of modal parameters is identifiable due to the combination of temporal and spatial information in a $z$ domain state vector of relatively high order (5-10). Since this $z$ domain state vector has both complex modal frequency and modal vector information as embedded content, sorting consistent estimates from the multitude of possible solutions is relatively trivial. Because this $z$ domain state vector can be developed from the results of any modal parameter estimation method, possible solutions from different traditional methods can be utilized in the autonomous procedure to yield one consistent set of modal parameters.
\end{abstract}

\section{Introduction}

The desire to estimate modal parameters automatically, once a set or multiple sets of test data are acquired, has been a subject of great interest for more than 40 years. In the 1960s, when modal testing was limited to analog test methods, several researchers were exploring the idea of an automated test procedure for determining modal parameters [1-3]. Today, with the increased memory and compute power of current computers used to process test data, an automated or autonomous, modal parameter estimation procedure is entirely possible and is being evaluated by numerous researchers and users.

Before proceeding with a discussion of the use and impact of spatial information in autonomous modal parameter estimation, some philosophy and definitions regarding what is considered autonomous is required. In general, autonomous modal parameter estimation refers to an automated procedure that is applied to a modal parameter estimation algorithm so that no user interaction is required once the process is initiated. This typically involves setting a number of parameters or thresholds that are used to guide the process in order to exclude solutions that are not acceptable to the user. When the procedure finishes, a set of modal parameters is identified that can then be reduced or expanded if necessary. The goal is that no further reduction, expansion, or interaction with the process will be required.

For the purposes of further discussion, the autonomous modal parameter estimation procedure is simply an efficient mechanism for sorting a very large number of solutions into a final set of solutions that satisfies a set of criteria and thresholds that are acceptable to the user. This user is assumed to be very experienced and uses autonomous modal parameter estimation as a sophisticated tool to highlight the most likely solutions based upon statistics. The experienced user will realize that the final solutions may include unrealistic solutions or nonoptimal solutions and further evaluation will be required. 


\section{Background}

In order to discuss the impact and use of spatial information in autonomous modal parameter estimation, some background is needed to clarify terminology and methodology. This background has been provided in previous papers and will only be highlighted here in terms of spatial information, modal parameter estimation, and autonomous modal parameter estimation.

2.1. Spatial Information. Spatial information, with respect to experimental modal parameter estimation, refers to the vector information and dimension associated with the inputs and outputs of the experimental test. Essentially, this represents the locations of the sensors in the experimental test. It is important to recognize that an experimental test should always include multiple inputs and outputs in order to clearly estimate different modal vectors and to resolve modal vectors when the complex natural frequencies are close, what is called repeated or pseudorepeated roots.

Since the data matrix, normally involving frequency response functions (FRF) or impulse response functions (IRF), is considered to be symmetric or reciprocal, the data matrix can be transposed, switching the effective meaning of the row and column index with respect to the physical inputs and outputs. Consider

$$
\left[H\left(\omega_{i}\right)\right]_{N_{o} \times N_{i}}=\left[H\left(\omega_{i}\right)\right]_{N_{i} \times N_{o}}^{T} .
$$

Since many modal parameter estimation algorithms are developed on the basis of either the number of inputs $\left(N_{i}\right)$ or the number of outputs $\left(N_{o}\right)$, assuming that one or the other is larger based upon test method, some nomenclature conventions are required for ease of further discussion. In terms of the modal parameter estimation algorithms, it is more important to recognize whether the algorithm develops the solution on the basis of the larger $\left(N_{L}\right)$ of $N_{i}$ or $N_{o}$ or the smaller $\left(N_{S}\right)$ of $N_{i}$ or $N_{o}$, dimension of the experimental data. For this reason, the terminology of long (larger of $N_{i}$ or $N_{o}$ ) dimension or short (smaller of $N_{i}$ or $N_{o}$ ) dimension is easier to understand without confusion.

Therefore, the nomenclature of the number of outputs $\left(N_{o}\right)$ and number of inputs $\left(N_{i}\right)$ has been replaced by the length of the long dimension of the data matrix $\left(N_{L}\right)$ and the length of the short dimension $\left(N_{S}\right)$ regardless of which dimension refers to the physical output or input. This means that the above reciprocity relationship can be restated as

$$
\left[H\left(\omega_{i}\right)\right]_{N_{L} \times N_{S}}=\left[H\left(\omega_{i}\right)\right]_{N_{S} \times N_{L}}^{T} .
$$

Note that the reciprocity relationships embedded in (1) and (2) are a function of the common degrees of freedom (DOFs) in the short and long dimensions. If there are no common DOFs, there are no reciprocity relationships and the data requirement for modern modal parameter estimation algorithms (multiple references) will not be met. Nevertheless, the importance of (1) and (2) is that the dimensions of the FRF matrix can be transposed as needed to fit the requirement of specific modal parameter estimation algorithms. This impacts the size of the square matrix coefficients in the matrix coefficient, polynomial equation, and the length of the associated modal (base) vector.

2.2. Modal Parameter Estimation. All modern, commercial algorithms for estimating modal parameters from experimental input-output data utilize matrix coefficient, polynomial models. This general matrix coefficient, polynomial formulation yields essentially the same polynomial form for both time and frequency domain data. Note, however, that this notation does not mean that, for an equivalent model order, the associated matrix coefficients are numerically equal.

For the frequency domain data case, this is

$$
\left|\left[\alpha_{m}\right] s^{m}+\left[\alpha_{m-1}\right] s^{m-1}+\left[\alpha_{m-2}\right] s^{m-2}+\cdots+\left[\alpha_{0}\right]\right|=0,
$$

where

$$
s_{r}=\lambda_{r}, \quad \lambda_{r}=\sigma_{r}+j \omega_{r} .
$$

For the time domain data case, this is

$$
\left|\left[\alpha_{m}\right] z^{m}+\left[\alpha_{m-1}\right] z^{m-1}+\left[\alpha_{m-2}\right] z^{m-2}+\cdots+\left[\alpha_{0}\right]\right|=0,
$$

where

$$
\begin{array}{cc}
z_{r}=e^{\lambda_{r} \Delta t}, \quad \lambda_{r}=\sigma_{r}+j \omega_{r}, \\
\sigma_{r}=\operatorname{Re}\left[\frac{\ln z_{r}}{\Delta t}\right], \quad \omega_{r}=\operatorname{Im}\left[\frac{\ln z_{r}}{\Delta t}\right] .
\end{array}
$$

The size of the square, matrix coefficients $([\alpha])$, and the order of the polynomial $(m)$ vary with the algorithm. Once the matrix coefficients $([\alpha])$ have been found, the modal frequencies $\left(\lambda_{r}\right.$ or $\left.z_{r}\right)$ can be found as the roots of the matrix coefficient polynomial (see (3) or (5)) using any one of a number of numerical techniques, normally involving an eigenvalue problem of the companion matrix associated with the matrix coefficient polynomial.

When the modal frequencies are estimated from this eigenvalue problem, a unique estimate of the unscaled modal vector is identified from the associated eigenvector at the same time. The length or dimension of this unscaled modal vector is equal to the dimension of the square, alpha coefficients which, in general, is equal to $N_{S}$ or $N_{L}$.

In all cases, the size of the square, alpha coefficient matrices $([\alpha])$, and/or the size of the model order $(m)$ can be varied to estimate multiple sets of solutions from the same data. These multiple sets of solutions are generally presented graphically in terms of consistency diagrams to identify a final set of realistic solutions. For this reason, with minor implementation differences, all of these algorithms can take advantage of the consistency diagram as an aid in identifying the correct complex modal frequencies from the large number of estimates that are found.

The most commonly used modal identification methods can be summarized as shown in Table 1 .

All of the methods summarized in Table 1 have a common characteristic in that they involve a matrix coefficient, characteristic polynomial of the form in (3) and (5). Once 
TABLE 1: Summary of modal parameter estimation algorithms.

\begin{tabular}{|c|c|c|c|c|c|c|c|}
\hline \multirow{2}{*}{ Algorithm } & \multicolumn{2}{|c|}{ Domain } & \multicolumn{3}{|c|}{ Matrix polynomial order } & \multicolumn{2}{|c|}{ Coefficients } \\
\hline & Time & Freq. & Zero & Low & High & Scalar & Matrix \\
\hline Complex Exponential Algorithm (CEA) & - & & & & • & • & \\
\hline Least Squares Complex Exponential (LSCE) & - & & & & - & - & \\
\hline Polyreference Time Domain (PTD) & - & & & & - & & $N_{S} \times N_{S}$ \\
\hline Ibrahim Time Domain (ITD) & • & & & - & & & $N_{L} \times N_{L}$ \\
\hline Multireference Ibrahim Time Domain (MRITD) & • & & & • & & & $N_{L} \times N_{L}$ \\
\hline Eigensystem Realization Algorithm (ERA) & $\bullet$ & & & • & & & $N_{L} \times N_{L}$ \\
\hline Polyreference Frequency Domain (PFD) & & - & & • & & & $N_{L} \times N_{L}$ \\
\hline Simultaneous Frequency Domain (SFD) & & - & & - & & & $N_{L} \times N_{L}$ \\
\hline Multireference Frequency Domain (MRFD) & & • & & • & & & $N_{L} \times N_{L}$ \\
\hline Rational Fraction Polynomial (RFP) & & $\bullet$ & & & - & - & $N_{S} \times N_{S}$ \\
\hline Orthogonal Polynomial (OP) & & $\bullet$ & & & • & $\bullet$ & $N_{S} \times N_{S}$ \\
\hline Polyreference Least Squares Complex Frequency (PLSCF) & & • & & & • & • & $N_{S} \times N_{S}$ \\
\hline Rational Fraction Polynomial-Z Domain (RFP-Z) & & - & & & - & - & $N_{S} \times N_{S}$ \\
\hline Complex Mode Indication Function (CMIF) & & - & • & & & & $N_{L} \times N_{S}$ \\
\hline
\end{tabular}

this is noted, all of these methods can be explained with a common mathematical form. In previous papers, this has been referred to as the Unified Matrix Polynomial Algorithm (UMPA) model [4-7]. Since both the frequency and time domain models are based upon functionally similar matrix coefficient, characteristic polynomials, the UMPA $(m, n, v)$ terminology can be used for models in both domains to reflect the order of the denominator polynomial $(m)$, the order of the numerator polynomial $(n)$, and the order of the base vector $(v)$ involved in the basic UMPA formulation. This common approach to all algorithms is particularly useful in autonomous modal parameter estimation procedures in that multiple, different algorithms can be more easily combined into a single solution approach. This is an extremely useful concept when developing autonomous modal parameter estimation procedures and bringing solutions from all methods together in one autonomous procedure. If the interested reader is unfamiliar with any of the modal parameter estimation algorithms cited in Table 1, the complete list of references for each modal parameter estimation algorithm can be found in one of the cited UMPA references [4-7].

Table 2 organizes the modal parameter estimation algorithms from Table 1 into one of four categories. Algorithms in each category have similar numerical structure and performance characteristics. The methods highlighted in bold font text in both tables are used together in a single autonomous modal parameter estimation procedure throughout the remainder of this paper. The highlighted methods are chosen as representative of the characteristics of all of the methods in their respective category in Table 2 and are representative of methods used by most practitioners in both research and commercial implementations.

As a matter of further clarification, two different Polyreference Frequency Domain (PFD) algorithms were developed independently in the 1980s. Both methods are low order, frequency domain methods. PFD-1 is the designation for the first order form and PFD-2 is the designation for the second
TABLE 2: Four corners of modal parameter estimation.

\begin{tabular}{lcc}
\hline & Time domain & Frequency domain \\
\hline \multirow{4}{*}{ Low order models } & & PFD-1 \\
& ITD & PFD-2 \\
& MRITD & PFD-Z \\
& ERA & SFD \\
& & MRFD \\
\hline \multirow{3}{*}{ High order models } & CEA & RFP \\
& LSCE & OP \\
& PTD & PFCF \\
& & PolyMAX \\
& & AF-Poly \\
\hline
\end{tabular}

order form. A recent paper explains that the two methods are theoretically equivalent [8].

2.3. Autonomous Modal Parameter Estimation. The interest in automatic modal parameter estimation methods has been documented in the literature since at least the mid-1960s when the primary modal method was the analog, force appropriation method [1-3]. Following that early work, there has been a continuing interest in autonomous methods that, in most cases, have been procedures that are formulated based upon a specific modal parameter estimation algorithm like the Eigensystem Realization Algorithm (ERA), the Polyreference Time Domain (PTD) algorithm, or more recently the Polyreference Least Squares Complex Frequency (PLSCF) algorithm or the commercial version of the PLSCF, the PolyMAX method [4-7]. A relatively complete list of autonomous and semiautonomous methods that have been reported prior to 2010 can be found in a recent paper [9]. The interested reader should also review other similar methods in more recent references [10-13].

Each of these past procedures has shown some promise but has not yet been widely adopted. In many cases, the procedure focussed on a single modal parameter estimation algorithm and did not develop a general procedure. Most of 
the past procedural methods focussed on modal frequency (pole) density but depended on limited modal vector data to identify correlated solutions. Currently, due to increased computational speed and availability of memory, procedural methods can be developed that were beyond the computational scope of available hardware only a few years ago. These methods do not require any initial thresholding of the solution sets and rely upon correlation of the vector space of hundreds or thousands of potential solutions as the primary identification tool.

The discussion in the following sections of the use and impact of spatial information in autonomous modal parameter estimation is based upon recent implementation and experience with an autonomous modal parameter estimation procedure referred to as the common statistical subspace autonomous mode identification (CSSAMI) method $[9,14-$ 16]. The strategy of the CSSAMI autonomous method is to use a default set of parameters and thresholds to allow for all possible solutions from a given data set. This strategy allows for some poor estimates to be identified as well as the good estimates. The philosophy of this approach is that it is easier for the user to evaluate and eliminate poor estimates compared to trying to find additional solutions. The reader is directed to a series of previous papers in order to get an overview of the methodology and to view application results for several cases $[9,14-16]$.

Note that much of the background of the CSSAMI method is based upon the Unified Matrix Polynomial Algorithm (UMPA) described in the previous section. This means that this method can be applied to both low and high order methods with short or long dimension modal (base) vectors. This also means that most commercial algorithms could take advantage of this procedure. Note that high order matrix coefficient polynomials normally have coefficient matrices of a dimension that is based upon the short dimension of the data matrix $\left(N_{S}\right)$. In these cases, it may be useful to solve for the complete, unscaled, or scaled, modal vector of the large dimension $\left(N_{L}\right)$. This will extend the temporal-spatial information in the modal (base) vector so that the vector will be more sensitive to change. This characteristic is what gives the CSSAMI autonomous method a robust ability to distinguish between computational and structural modal parameters.

\section{Pole-Weighted Modal Vectors}

When comparing modal (base) vectors, at either the short or the long dimension, a pole-weighted vector can be constructed independent of the original algorithm used to estimate the poles and modal (base) vectors. For a given order $k$ of the pole-weighted vector, the modal (base) vector and the associated pole can be used to formulate the pole-weighted vector as follows:

$$
\{\phi\}_{r}=\left\{\begin{array}{c}
\lambda_{r}^{k}\{\psi\}_{r} \\
\vdots \\
\lambda_{r}^{2}\{\psi\}_{r} \\
\lambda_{r}^{1}\{\psi\}_{r} \\
\lambda_{r}^{0}\{\psi\}_{r}
\end{array}\right\}_{r}, \quad\{\phi\}_{r}=\left\{\begin{array}{c}
z_{r}^{k}\{\psi\}_{r} \\
\vdots \\
z_{r}^{2}\{\psi\}_{r} \\
z_{r}^{1}\{\psi\}_{r} \\
z_{r}^{0}\{\psi\}_{r}
\end{array}\right\}_{r}
$$

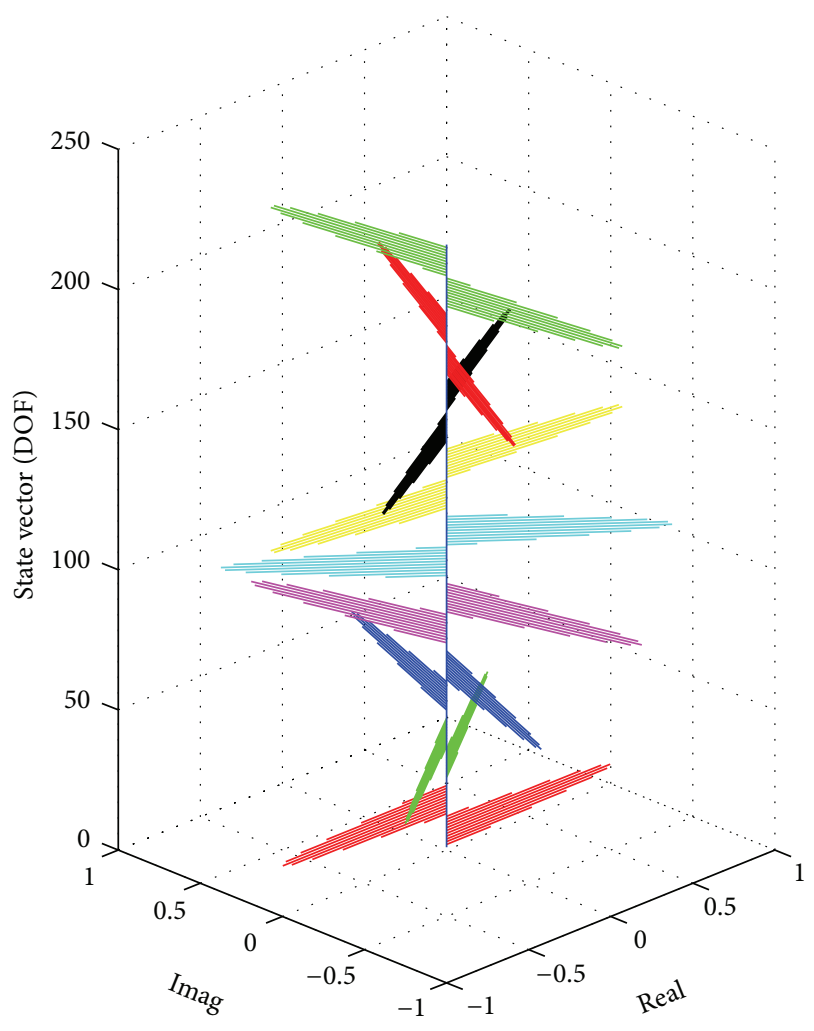

FIGURE 1: Eighth order, pole-weighted vector (state vector) example.

While the above formulation (on the left) is possible, this form would be dominated by the high order terms if actual frequency units are utilized. Generalized frequency concepts (frequency normalization or $z$ domain mapping) are normally used to minimize this issue by using the $z$ domain form $\left(z_{r}\right)$ of the complex modal frequency $\left(\lambda_{r}\right)$ as shown above (on the right). The $z$ domain form of the complex natural frequency is developed as follows:

$$
\begin{gathered}
z_{r}=e^{\pi *\left(\lambda_{r} / \Omega_{\max }\right)}, \\
z_{r}^{m}=e^{m * \pi *\left(\lambda_{r} / \Omega_{\max }\right)} .
\end{gathered}
$$

In the above equations, $\Omega_{\max }$ can be chosen as needed to cause the positive and negative roots to wrap around the unit circle in the $z$ domain without overlapping (aliasing). Normally, $\Omega_{\max }$ is taken to be five percent larger than the largest frequency (absolute value of the complex frequency) identified in the roots of the matrix coefficient polynomial.

Figures 1 and 2 are graphical representations of the pole-weighted vector (state vector) defined in (7). In this representative example, the modal (base) vector (at the bottom of Figure 1) is a real-valued, normal mode that looks like one period of a sine wave. The successive higher orders, up to order eight, are shown in different colors moving up the vertical axis of this figure. The effect of scaling of the modal (base) vector by the higher powers of the $z$ domain frequency value causes the base vector to rotate in the real and imaginary space. Figure 2 shows the rotation effect clearly. Note that the choice of the order $(k)$ of the pole-weighted 


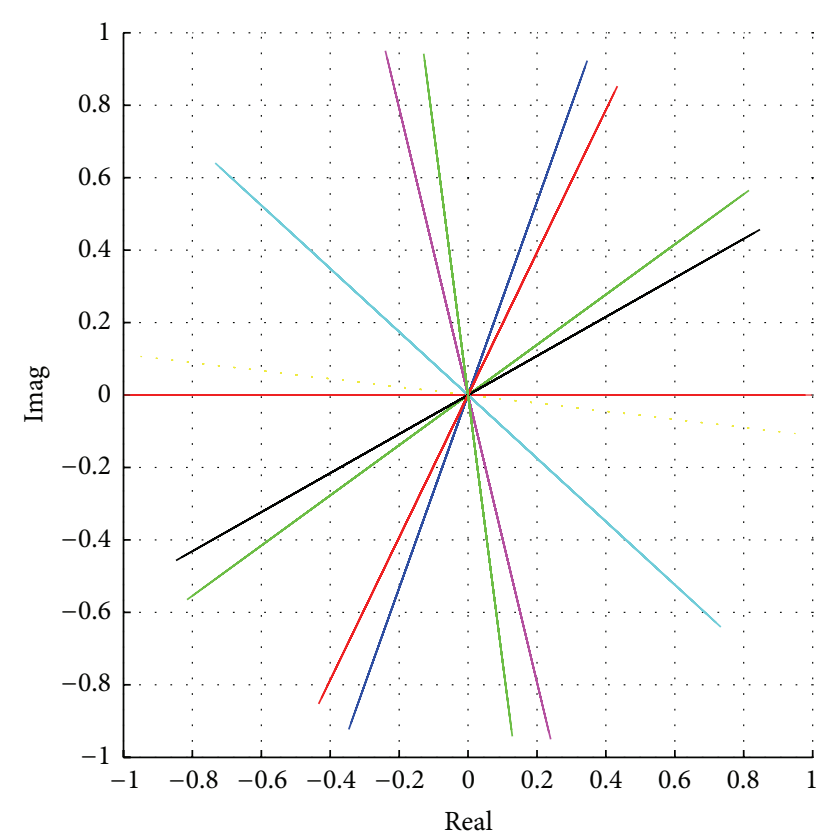

FIGURE 2: Eighth order, pole-weighted vector (state vector) example, top view.

vector, therefore, just generates additional length and rotation in the pole-weighted vector and gives varying sensitivity to comparisons between estimates. Furthermore, note that the choice of order $(k)$ is independent of the modal parameter estimation algorithm and the order of the matrix polynomial used in the algorithm. State vectors are a natural part of the numerical formulation for all modal parameter estimation algorithms, but this pole-weighted vector (state vector) which looks similar is not constrained by the algorithm. Note that if the base vector is complex-valued, rather than real-valued, each vector contribution to the pole-weighted vector will contain this real versus imaginary scatter and Figure 2 will present rotated, banded areas rather than the rotated lines in this example.

The order $(k)$ of the pole-weighted vector (state vector) is up to the user. Generally, if spatial dimension used to generate the pole-weighted vector $\left(N_{S}\right.$ and/or $\left.N_{L}\right)$ is small, a higher order will be required to discriminate between modal vectors that have similar spatial characteristics, maybe as high as 50 or 60 . As the spatial dimension gets bigger, the model order $(k)$ can be chosen more modestly. Essentially, the order $(k)$ of the pole-weighted vector is inversely proportional to the dimension of the base vector. Experience has shown that an order of four or five is probably the minimum and that larger choices for model order $(k)$ do not appear to cause any issues. For the purposes of the examples used in this paper, the order has been chosen to be ten, meaning orders of zero to ten (eleven times the base vector) have been used to generate the pole-weighted vectors.

While the order $(k)$ of the pole-weighted vector (state vector) can be raised ( $k=50$ or $k=60$, e.g.) for a case with minimal base vector dimension (two or three, e.g.), this is generally not as desirable as using a larger base vector (thirty, e.g.) raised to a more modest order $(k=5$ or $k=6$, e.g.). In this example, the longer base vector would have more independent spatial information contributing to the pole-weighted vector. While this is intuitive and has been observed to be the case, no formal study has taken place concerning this issue. Using the longest base vector seems to be the prudent approach, assuming that the data is representative of the DOF space.

Since the magnitude of the $z$ domain frequency value is unity, there is no magnitude weighting involved. This rotation gives a method for a single vector to represent the modal (base) vector shape together with the complex-valued frequency. With respect to sorting and separating modal vectors that have similar shapes but different frequencies or similar frequencies but different modal vector shapes, this becomes a powerful parameter, together with modal vector correlation tools like the modal assurance criterion (MAC) $[17,18]$, for modal parameter estimation and for autonomous modal parameter estimation.

\section{Multialgorithm, Extended Consistency Diagrams}

Consistency diagrams have historically been developed for a specific modal parameter estimation algorithm. As such the numerical implementation can be different as a function of basis dimension $\left(N_{S}\right.$ or $\left.N_{L}\right)$, model order, and/or subspace iteration. This would make it very hard to combine different algorithms into a single consistency diagram. However, every algorithm, at the point of the numerical implementation of the consistency diagram, has multiple sets of complex modal frequency and complex-valued modal vectors. The modal vectors may be of different length $\left(N_{S}\right.$ or $\left.N_{L}\right)$ as a function of algorithm. This potential mismatch in modal (base) vector length can be solved by restricting the long dimension to the DOFs of the short dimension or, more preferably, adding an extra step in the solution procedure to estimate the missing portion of the short dimension vectors, extending them to the long dimension DOFs. The latter approach is used in the following two figures as an example of extended consistency diagrams based upon multiple modal parameter estimation algorithms. In these examples, the results from the individual algorithms are simply stacked into the extended consistency diagram with common sorting and evaluation settings.

The data used for this, and all following examples in this paper, is FRF data taken from an impact test of a steel disc supported in a pseudo-free-free boundary condition. The steel disc is approximately $2 \mathrm{~cm}$. thick and $86 \mathrm{~cm}$. in diameter with several small holes through the disc. The center area of the disc (diameter of approximately $25 \mathrm{~cm}$.) has a thickness of approximately $6 \mathrm{~cm}$. There are seven reference accelerometers and measured force inputs from an impact hammer are applied to thirty-six locations, including next to the seven reference accelerometers. The frequency resolution of the data is 5 Hertz. While the disc is not as challenging as some industrial data situations that contain more noise or other complicating factors like small nonlinearities, the disc has a number of pseudorepeated roots spaced well within the $5 \mathrm{Hertz}$ frequency resolution and a mix of close modes involving repeated and nonrepeated roots which are very 


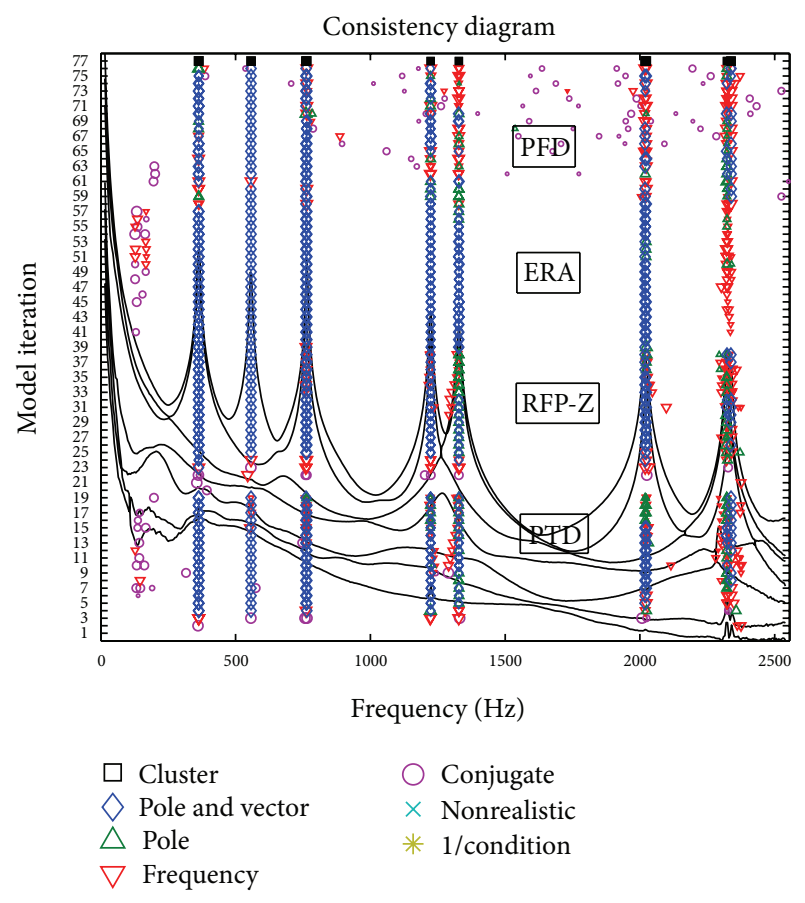

FIGURE 3: Extended consistency diagram, conventional version.

challenging. Based upon the construction of the disc, realvalued, normal modes can be expected and the inability to resolve these modes can be instructive relative to both modal parameter estimation algorithm and autonomous procedure performance. For the interested reader, a number of realistic examples are shown in other past papers including FRF data from an automotive structure and a bridge structure $[9,16]$.

Figure 3 is an example of using a conventional, sequential sorting procedure involving criteria for frequency, damping, and modal vector consistency. The symbol designations in the legend box identify consistency based upon separate user definitions regarding frequency; frequency and damping (pole); and frequency, damping, and modal vector closeness. Modal vector consistency is determined by using typical vector correlation methods (MAC) $[17,18]$ to identify consistency. In this type of consistency diagram, the only consistency comparisons that are made compare the current model order values to the previous model order values. This sequential estimation of consistency allows for some parameter drift as model order or algorithm is changed.

The figure includes a complex mode indicator function (CMIF) for the FRF dataset in the background. The blue diamond symbols indicate that modes have been identified with consistent frequency, damping and modal vector estimates as both model order and algorithm are changed. From the CMIF plot in the background, it is clear that most of the identified modes are closely spaced, repeated roots. For these cases, there are two blue diamonds at each solution on top of one another. The multialgorithm approach is sensitive to the transitions between algorithms as the modal parameter estimates and symbol designations are estimated sequentially across these transitions.

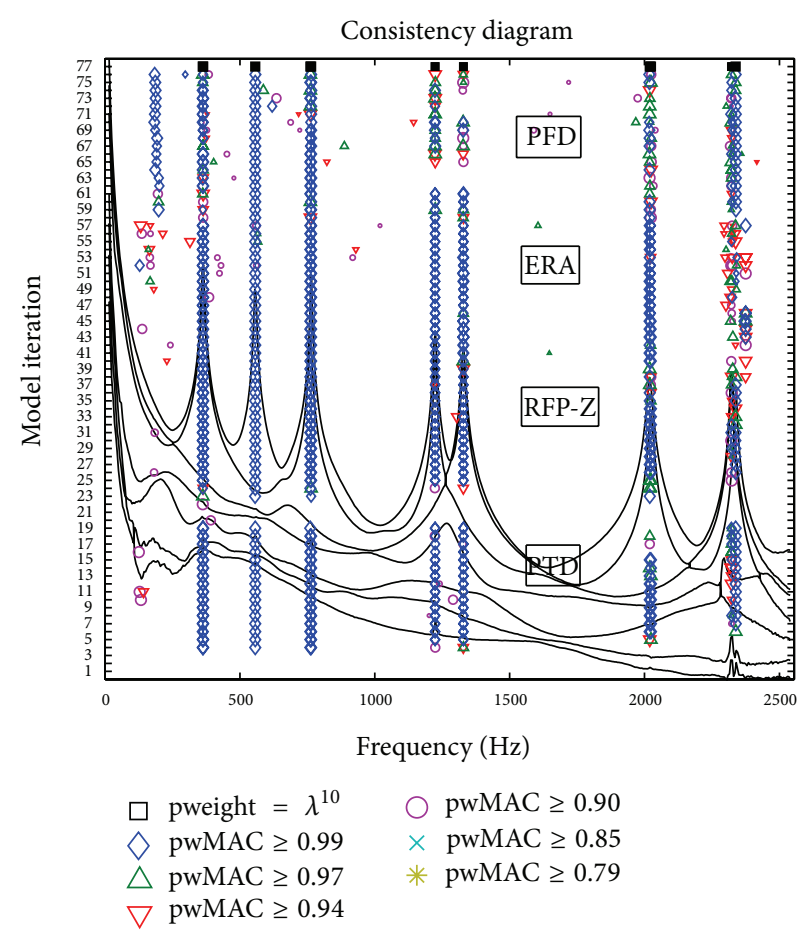

FIGURE 4: Extended consistency diagram, pole-weighted MAC version.

Figure 4 is an example using a pole-weighted vector (state vector) method of producing a similar consistency diagram. This approach is easier to implement numerically and provides equal or better results when compared to the conventional approach for almost all cases. In this example, every estimate from every matrix coefficient polynomial solution from every algorithm is converted into a poleweighted vector of a specific order, in this case tenth order. Then, the consistency diagram is developed by using the same vector correlation methods (MAC) $[17,18]$ to identify consistency without a need for a separate consistency comparison for frequency and damping (since the modal frequency is included in the pole-weighted vector). A similar set of symbols, as those used in Figure 3, are used to define increased levels of vector consistency in terms of the MAC value between all pole-weighted vectors.

Both methods work very well but the implementation of Figure 4 is computationally easier and not subject to a frequency drift in the symbol path that can occur in the conventional implementation, shown in Figure 3, that is due to frequency and damping variation with model order for each algorithm. Note that the solid square symbols at the top of both consistency diagrams represent the solution found from the CSSAMI autonomous modal parameter estimation procedure applied to the information represented by each consistency diagram.

\section{Autonomous Modal Parameter Estimation with Extended Consistency Diagrams}

The CSSAMI autonomous procedure utilizes all solutions indicated by a symbol in the consistency diagram. If some 


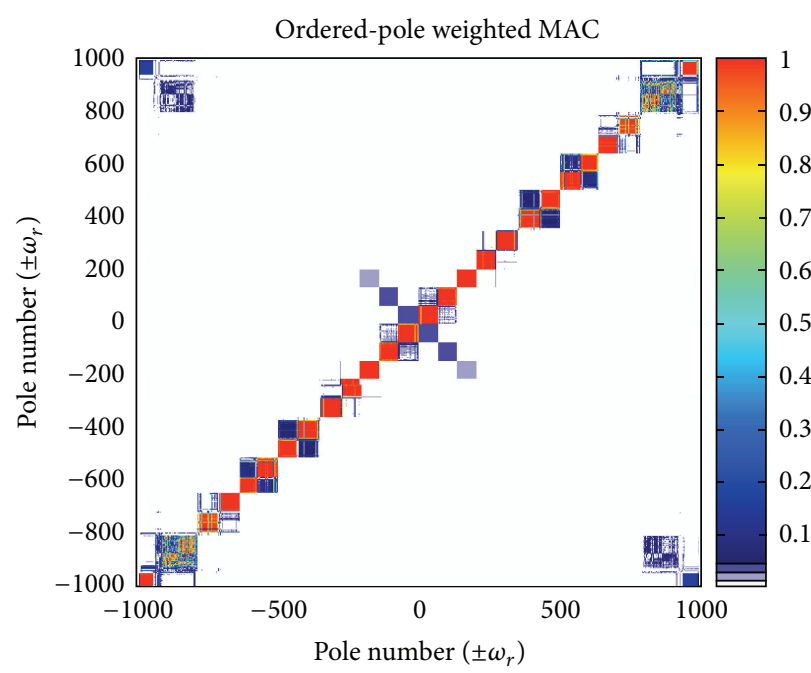

(a)

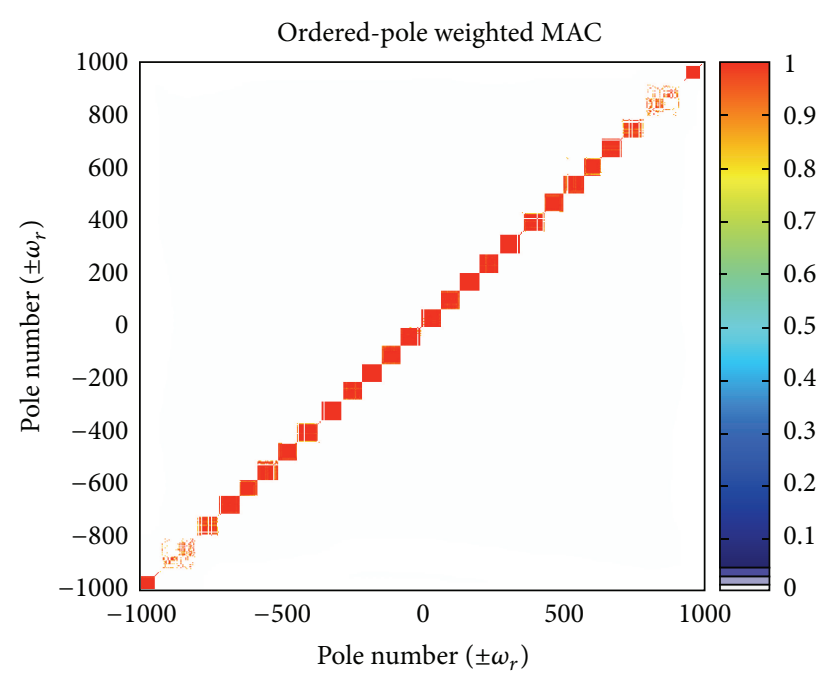

(b)

FIgURE 5: Pole-weighted MAC of all consistency diagram solutions, before and after threshold applied.

symbols are not present, it means that the user has decided not to view solutions identified by those symbols. This provides a way to remove solutions from the autonomous procedure that are clearly not reasonable. However, experience with the CSSAMI autonomous procedure has shown that some solutions that are often eliminated by users in an attempt to have a clear consistency diagram are often statistically consistent and useful.

Figure 5 shows the solutions that are included in the autonomous procedure. The graphical representation on the left represents an auto-MAC matrix involving the poleweighted vectors for all possible solutions from Figure 3 compared to themselves. Naturally, the diagonal will be 1.0 but when the off-diagonal terms are 1.0 or nearly 1.0, this means that the pole-weighted vectors are consistent and represent a cluster. The graphical representation on the right represents the pole-weighted vectors that remain after a MAC threshold (0.9) and minimum cluster size (4) limitations are imposed. Each cluster that remains is evaluated, cluster by cluster, independently to estimate the best modal frequency and modal vector from that cluster. Note that both the positive frequency and negative frequency (complex conjugate) roots are included and identified separately as clusters. Figure 5 represents nearly 1000 solution estimates spanning four different algorithms and 19 different solutions from each algorithm.

For the examples used in this paper, a minimum cluster size is chosen to be four. This choice is up to the user and generally should not be chosen too large. The philosophy is to allow the autonomous procedure to identify all possible clusters and then have the experienced user make the final decision if some of the clusters yield nonphysical results. These decisions can be made based upon statistics associated with the cluster or physical characteristics of the modal parameters. If the cluster size is too small, some modal vectors might be excluded. This would require the user to reprocess the data looking for a missing modal vector. It is far easier for the user to remove unwanted or nonphysical modes than to try to find modes that have been marginally excited in the data. The first column of Table 3 shows the cluster size for each mode estimated from the autonomous procedure.

Once the final set of modal parameters, along with their associated statistics, is obtained, quality can be assessed by many methods that have been used in the past. The most common example is to perform comparisons between the original measurements and measurements synthesized from the modal parameters. Another common example is to look at physical characteristics of the identified parameters such as reasonableness of frequency and damping values, normal mode characteristics in the modal vectors, and appropriate magnitude and phasing in the modal scaling. Other evaluations that may be helpful are modal assurance criterion (MAC) evaluation of the independence of the complete modal vector set, mean phase correlation (MPC) of each vector, or any other method available. Naturally, since a significant number of pole-weighted vectors are used in a cluster to identify the final modal parameters, traditional statistics involving mean and standard deviation are now available. Some of these statistical methods are discussed in the next section.

\section{Statistical Evaluation Parameters}

Statistical evaluation parameters can be estimated for each common cluster of pole-weighted vectors on the basis of the complex modal frequency, the modal vector, and the modal scaling. The number of pole-weighted vectors will in general be different in each cluster so the statistics will be based upon the number of estimates available (sample size $N$ ). Examples of the statistics currently computed for each modal parameter are described in the following sections.

6.1. Modal Frequency Statistics. The weighted modal frequency for the cluster is found by constructing the poleweighted vector (typically 10th order) for each pole retained 
TABLE 3: Summary of autonomous modal parameter estimation statistics.

\begin{tabular}{lccccccccccc}
\hline$N$ & Freq. (SV) & Damp (SV) & $\%$ Zeta & MPC & Freq. (Mean) & Damp (Mean) & Std. Dev. & NMVR $_{1} \%$ & NMVR $_{2} \%$ & NSVR $_{1} \%$ & NSVR $_{2} \%$ \\
\hline 69 & 362.356 & -3.128 & 0.8633 & 0.9199 & 362.356 & -3.128 & 0.1049 & 0.7686 & 6.3402 & 0.7686 & 6.3402 \\
69 & 363.696 & -3.403 & 0.9358 & 0.8915 & 363.696 & -3.403 & 0.1112 & 1.2308 & 10.2036 & 1.2312 & 10.2062 \\
71 & 557.008 & -2.888 & 0.5184 & 0.9926 & 557.008 & -2.888 & 0.1144 & 0.0810 & 0.6281 & 0.0812 & 0.6281 \\
69 & 761.172 & -5.091 & 0.6688 & 0.9701 & 761.172 & -5.091 & 0.1345 & 0.8888 & 7.1128 & 0.8889 & 7.1129 \\
70 & 764.175 & -2.581 & 0.3377 & 0.9862 & 764.175 & -2.581 & 0.0865 & 0.3335 & 2.2550 & 0.3337 & 2.2566 \\
64 & 1222.940 & -4.074 & 0.3332 & 0.8286 & 1222.940 & -4.074 & 0.0855 & 0.9708 & 6.4450 & 0.9712 & 6.4492 \\
67 & 1224.080 & -3.962 & 0.3236 & 0.8316 & 1224.079 & -3.962 & 0.1645 & 1.0343 & 8.3785 & 1.0338 & 8.3748 \\
68 & 1327.955 & -6.704 & 0.5048 & 0.8264 & 1327.953 & -6.705 & 0.3577 & 1.2250 & 9.9197 & 1.2248 & 9.9176 \\
57 & 1328.699 & -5.421 & 0.4080 & 0.8169 & 1328.698 & -5.421 & 0.1400 & 0.8649 & 6.3990 & 0.8645 & 6.3960 \\
69 & 2019.161 & -8.153 & 0.4038 & 0.9655 & 2019.163 & -8.159 & 1.0262 & 1.0610 & 7.3389 & 1.0623 & 7.3411 \\
56 & 2023.589 & -7.525 & 0.3719 & 0.9481 & 2023.590 & -7.525 & 0.5596 & 1.8525 & 11.7555 & 1.8518 & 11.7490 \\
18 & 2321.822 & -3.947 & 0.1697 & 0.7848 & 2321.819 & -3.947 & 0.5460 & 3.3706 & 14.0340 & 3.3657 & 14.0137 \\
4 & 2324.280 & -3.537 & 0.1522 & 0.8555 & 2324.271 & -3.537 & 1.3115 & 7.3179 & 13.3856 & 7.3251 & 13.3951 \\
47 & 2337.946 & -4.194 & 0.1794 & 0.9654 & 2337.945 & -4.201 & 0.9058 & 0.9132 & 4.5838 & 0.9129 & 4.5811 \\
\hline
\end{tabular}

in a cluster, then taking the SVD of the group of poleweighted vectors and selecting the singular vector associated with the largest singular value. This chosen singular vector contains both the shape and the modal frequency information. The modal frequency is identified by dividing the first order portion by the zeroth order portion of the vector in a least squares sense. (Note that it is also possible to solve the frequency polynomial which would result from using the complete vector.) Also, for numerical reasons, the poleweighted vector is actually computed in the $z$ domain.

For comparison purposes, the actual mean value and standard deviation of the poles (as well as the separate frequency and damping means and standard deviations) which were used in the computation of the weighted solution are computed. Since these results are unweighted by the vector characteristics, they may be somewhat different from the vector weighted solution and provide comparative feedback about the pole.

6.2. Modal Vector Statistics. In order to evaluate the quality of the resulting modal vectors, several different parameters (representing noise to signal ratios) are calculated. These ratios are evaluated for both the original normalized vectors and the pole-weighted (state extended) vectors and are computed using the singular value decomposition of each of the set of vectors.

6.2.1. Normalized Modal Vector Residual (NMVR). The first modal vector parameter is evaluated by taking the total residual magnitude (the Frobenius norm of the residuals) divided by the magnitude of the principal vector magnitude. In other words, the square root of the sum of the squares of the residual singular values divides by the first (largest) singular value. This provides an indication of the consistency of the original contributing vectors. Small values tend to indicate greater consistency. Large values indicate greater variance or the possibility that more than one mode has been included in a cluster. Consider

$$
\begin{aligned}
& \lceil\sigma\rfloor=\operatorname{SVD}\left(\left[\psi_{1} \psi_{2} \cdots \psi_{N}\right]\right), \\
& \mathrm{NMVR}_{1}=\frac{1}{\sigma_{1}}\left(\frac{\sum_{k=2}^{N} \sigma_{k}^{2}}{N}\right)^{1 / 2}
\end{aligned}
$$

The second modal vector parameter is evaluated by taking the largest residual magnitude divided by the magnitude of the principal vector magnitude. In other words, the second singular value divided by the first singular value. This provides an indication of the consistency of the original contributing vectors. A small value tends to indicate random variance. A larger value can indicate a consistent modal contamination of the original vectors, possibly caused by a second mode included in the cluster:

$$
\mathrm{NMVR}_{2}=\frac{\sigma_{2}}{\sigma_{1}} .
$$

6.2.2. Normalized State Vector Residual (NSVR). The associated state vector parameters are calculated analogous to the above except that the complete pole-weighted vector (state vector) is used:

$$
\begin{gathered}
\lceil\sigma\rfloor=\operatorname{SVD}\left(\left[\phi_{1} \phi_{2} \cdots \phi_{N}\right]\right), \\
\operatorname{NMVR}_{1}=\frac{1}{\sigma_{1}}\left(\frac{\sum_{k=2}^{N} \sigma_{k}^{2}}{N}\right)^{1 / 2}, \\
\operatorname{NSVR}_{2}=\frac{\sigma_{2}}{\sigma_{1}}
\end{gathered}
$$

6.3. Modal Scaling Statistics. If modal scaling (residue) information is available in the original vectors, it can be 
applied to the principal pole-weighted vector such that the consistency of modal scaling (Modal A) can also be evaluated. By scaling the largest driving point response to unity, a uniform, comparable value for Modal A is chosen. Since the modal scaling parameter (Modal A) contains the physical units and hence the relative contribution of each pole/vector combination to the original data set, a means of evaluating the significance and confidence in each estimate is possible. Because the original vectors represent multiple scaled solutions and since Modal $\mathrm{A}$ is a single scalar complex value for each pole/vector combination, the statistical distribution of Modal A (mean and standard deviation) of the original cluster can be compared with the scaling estimated from the pole-weighted (state extended) vector. The magnitude of Modal A can be used to evaluate the contribution of this vector to the total set of identified vectors. When the user believes that the identified vectors should be normal modes, the phase of the Modal A can be used to evaluate the closeness to a normal mode.

6.4. Example: Statistical Evaluation. Table 3 represents a tabulation of the modal parameters found along with some of the statistical information described in the previous sections. Most of the indicators are quite good with the exception of the modes in the region around 2320 Hertz. The fact that the size of the clusters is much smaller (18 and 4) than all of the other clusters is a quick way to identify possible problems.

Figure 6 shows the location of the complex modal frequency estimates in the second quadrant of the $S$ plane. The scatter in the modal frequency estimates is clearly identifiable. However, the exact cause of the scatter is not definitive.

The two modes in this region, within one $\Delta f$, plus a third mode within four $\Delta f$, are providing a difficult identification problem. The spatial resolution of the sensors is also nearly at a limit due to the complexity of the motion in the modal vector. This contamination of the modal vectors, when resolving close modal frequencies, will be discussed briefly in the next section using some variations of the modal assurance criterion (MAC).

\section{Future Work: Resolving Modal Vector Contamination}

Much of the CSSAMI autonomous procedure, as well as most commonly used modal parameter estimation methods, utilize the traditional modal assurance criterion (MAC) computation, restated in (12), to sort the numerous possible solutions into clusters related to different modes or to identify contamination between modal vectors:

$$
\operatorname{MAC}_{c d}=\frac{\left|\left\{\psi_{c}\right\}^{H}\left\{\psi_{d}\right\}\right|^{2}}{\left\{\psi_{c}\right\}^{H}\left\{\psi_{c}\right\}\left\{\psi_{d}\right\}^{H}\left\{\psi_{d}\right\}}=\frac{\left\{\psi_{c}\right\}^{H}\left\{\psi_{d}\right\}\left\{\psi_{d}\right\}^{H}\left\{\psi_{c}\right\}}{\left\{\psi_{c}\right\}^{H}\left\{\psi_{c}\right\}\left\{\psi_{d}\right\}^{H}\left\{\psi_{d}\right\}} .
$$

The only difference in the CSSAMI autonomous modal parameter estimation procedure is that the pole-weighted vectors of an arbitrarily (user defined) order are constructed and used in the MAC calculation so that the dimension

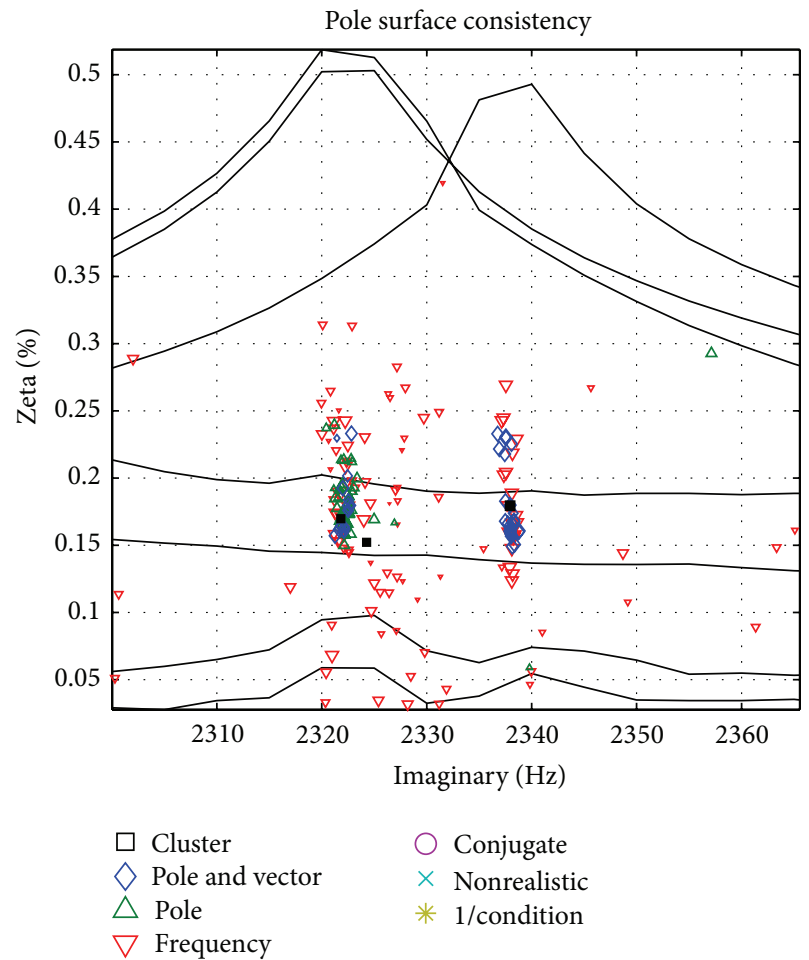

FIGURE 6: Pole surface consistency, 2320 Hertz region.

of each vector is much larger. In this way, the clusters for spatially undersampled modal vectors can be separated from one another. Likewise, modal vectors associated with close frequencies will be separated into distinct clusters. This is done automatically once (1) the order of the pole-weighted vector is chosen and (2) the lowest acceptable threshold for MAC correlation is chosen. A third parameter is also needed (3) to define the minimum number of modal vector estimates required to define a cluster (otherwise clusters of one or two will be identified that generally represent spurious estimates based upon noise on the data). Normally this minimum number of modal vectors to define a cluster is greater when more estimates are available, as in the case where multiple modal parameter estimation algorithms are incorporated into the autonomous procedure. Figure 5 is a graphical representation of the before and after information gained from this MAC procedure.

Note that the CSSAMI autonomous procedure treats each cluster of estimates independently and does not constrain the final set of modal vector estimates in any way. At this point in the autonomous procedure, the MAC computation is again utilized to evaluate the quality of the solutions. This begins with an evaluation of the MAC between all of the modal vectors in the final set to ascertain whether the modal set is an independent set of vectors. This often involves including the estimates of the modal vectors associated with the conjugate poles. Since the conjugate poles and vectors are estimated separately, if nonconjugate relationships exist between the associated modal vector estimates (between the modal vector for pole and the modal vector of the conjugate 
pole), the MAC between these two associated vectors will not be unity as expected. A number of users have noted that this often correlates with modal vectors that are exhibiting some unexpected, nonphysical characteristics.

Since much of the autonomous procedure is based upon numerical processing methods like singular value decomposition (SVD), solutions that are identified, based upon the data associated with a cluster of estimates, have no physical or causal constraint. An example of a physical or causal constraint would be the expectation of real-valued, normal modes for systems where no expectation of nonproportional damping is likely. SVD methods will identify the most dominant unitary (orthogonal and unit length) vectors in a cluster, yielding a complex-valued vector. Experience has shown that when modes are very close in frequency with minimal spatial resolution, the complex-valued vectors will still show significant dependence or correlation.

However, when these complex-valued vectors are examined closely, the nondominant portion of the complex-valued vector often correlates very highly with one or more nearby modal vectors. This can be examined by the MAC calculation or several variants of the MAC calculation (like the weighted MAC calculation defined in (14)). Identifying the potential contamination of modal vectors is helpful to the thorough understanding of the modal parameter estimation algorithms and autonomous procedure as well as being instructive for potential removal of the contamination. If some sort of real normalization is desirable (to match up well with an undamped analytical model, e.g.), understanding of the contamination that is being removed is a prerequisite to any procedure. Random contamination may simply be ignored, smoothed, or averaged out, but if the contamination is related to nearby modes, it may indicate that the modal parameter estimation may need further evaluation or that more data is required.

For this evaluation of the modal vector contamination, it will be easiest to first rotate each complex-valued modal vector to a real (or imaginary) dominant vector. This is done by using a least squares method to identify the rotation of the modal vector away from the real or imaginary axis and then using the associated complex phasor to rotate each original complex-valued modal vector to a new complexvalued modal vector that aligns with the real or imaginary axis. For all following discussions, the original complexvalued modal vectors are rotated to be dominantly realvalued. It is convenient, for display reasons, to also normalize the new complex-valued modal vector to a unity maximum or unity vector length. Naturally, this rotation and rescaling must be considered in any final estimates of modal scaling.

To understand the nature of the possible modal vector contamination in a complex-valued modal vector, three conventional MAC calculations can be performed (1) between the real parts of the modal vectors and the complex-valued modal vectors (rMAC), (2) between the imaginary parts of the modal vectors and the complex-valued modal vectors (iMAC), and (3) between the real parts of the modal vectors and the imaginary parts of the modal vectors (riMAC). These three MAC calculations and the interpretation of these MAC values will be sensitive to the rotation and normalization of the complex-valued modal vector estimates. The following use and discussion assumes that the complex-valued modal vectors have been rotated so that the central axis of the complex-valued modal vector is centered on the real axis. These three MAC computations identify (1) that the real part of the modal vector is the dominant part of the complexvalued modal vector (rMAC), (2) that the imaginary part of the modal vector is the dominant part of the complex-valued modal vector (iMAC), and (3) that the real and imaginary parts of the modal vector are, or not, related to one another. All MAC computations in this case are, as always, bounded from zero to one. If near normal modes are expected, (1) the rMAC should be close to one, (2) the iMAC should be close to zero, and (3) the riMAC should also be close to zero. Note in the following definitions, complex-valued modal vectors $c$ and $d$ can again be any of the modal vectors that the user wishes to include in the evaluation:

$$
\begin{aligned}
& \operatorname{rMAC}_{c d}=\frac{\left(\operatorname{Re}\left\{\psi_{c}\right\}^{H}\right)\left\{\psi_{d}\right\}\left\{\psi_{d}\right\}^{H}\left(\operatorname{Re}\left\{\psi_{c}\right\}\right)}{\left(\operatorname{Re}\left\{\psi_{c}\right\}^{H}\right)\left(\operatorname{Re}\left\{\psi_{c}\right\}\right)\left\{\psi_{d}\right\}^{H}\left\{\psi_{d}\right\}} \\
& \mathrm{iMAC}_{c d}=\frac{\left(\operatorname{Im}\left\{\psi_{c}\right\}^{H}\right)\left\{\psi_{d}\right\}\left\{\psi_{d}\right\}^{H}\left(\operatorname{Im}\left\{\psi_{c}\right\}\right)}{\left(\operatorname{Im}\left\{\psi_{c}\right\}^{H}\right)\left(\operatorname{Im}\left\{\psi_{c}\right\}\right)\left\{\psi_{d}\right\}^{H}\left\{\psi_{d}\right\}}, \\
& \operatorname{riMAC}_{c d}=\frac{\left(\operatorname{Re}\left\{\psi_{c}\right\}^{H}\right)\left(\operatorname{Im}\left\{\psi_{d}\right\}\right)\left(\operatorname{Im}\left\{\psi_{d}\right\}^{H}\right)\left(\operatorname{Re}\left\{\psi_{c}\right\}\right)}{\left(\operatorname{Re}\left\{\psi_{c}\right\}^{H}\right)\left(\operatorname{Re}\left\{\psi_{c}\right\}\right)\left(\operatorname{Im}\left\{\psi_{d}\right\}^{H}\right)\left(\operatorname{Im}\left\{\psi_{d}\right\}\right)}
\end{aligned}
$$

Use of the above modified MAC evaluations indicate that the imaginary part (contamination) of a given mode is strongly related to the real part (dominant) of the modal vector associated with its pseudorepeated root companion. This is consistent with theory that explains the cause of a complexvalued modal vector when two real-valued modal vectors are close in frequency and misidentified as a single modal vector [19].

The above MAC evaluations identify whether, and how, the contamination of a complex-valued modal vector is related to another of the identified modal vectors. However, the MAC computation is normalized to vector length, vector by vector, for the vectors used in the calculation. A weighted MAC can be used to determine the scale of the contamination. The following three definitions of the weighting for each of the above MAC calculations limit the associated MAC value to a fraction of the zero to one scale. If near normal modes are expected, (1) the weighting and rwMAC should be close to one, (2) the weighting and iwMAC should be close to zero, and (3) the combined weighting and riwMAC should also be close to zero. Note in the following definitions, complex-valued modal vectors $c$ and $d$ can again be any of the modal vectors that the user wishes to include in the evaluation:

$$
\begin{aligned}
& \operatorname{rwMAC}_{c d}=r W_{c} \times \mathrm{rMAC}_{c d} \\
& \text { where } r W_{c}=\frac{\left(\operatorname{Re}\left\{\psi_{c}\right\}^{H}\right)\left(\operatorname{Re}\left\{\psi_{c}\right\}\right)}{\left\{\psi_{c}\right\}^{H}\left\{\psi_{c}\right\}},
\end{aligned}
$$




$$
\begin{aligned}
\operatorname{iwMAC}_{c d} & =i W_{c} \times \mathrm{iMAC}_{c d} \\
\text { where } i W_{c} & =\frac{\left(\operatorname{Im}\left\{\psi_{c}\right\}^{H}\right)\left(\operatorname{Im}\left\{\psi_{c}\right\}\right)}{\left\{\psi_{c}\right\}^{H}\left\{\psi_{c}\right\}}, \\
\text { riwMAC }_{c d} & =r W_{c} \times i W_{d} \times \mathrm{riMAC}_{c d} .
\end{aligned}
$$

At this point, now that the contamination of the complexvalued modal vectors can be confirmed to be from the dominant portion (real part) of other complex-valued modal vectors and that the contamination is not significant, a strategy for determining the best set of real-valued modal vectors can be identified. One reasonable option would be to place the real parts and imaginary parts of each complexvalued modal vector into a matrix as separate real-valued vectors. A singular value decomposition of this real-valued matrix will yield real-valued singular vectors and the most significant singular vectors, equal to the original number of complex-valued modal vectors, associated with the largest singular values can be utilized as the final set of real-valued, normal modes. A simpler solution would be to eliminate the imaginary parts since the scale of the contamination is shown to be small.

\section{Summary}

With the advent of more computationally powerful computers and sufficient memory, it has become practical to evaluate sets of solutions involving hundreds or thousands of modal parameter estimates and to extract the common information from those sets. In many cases, autonomous procedures give very acceptable results, in some cases superior results, in a fraction of the time required for an experienced user to get the same result. However, it is important to reiterate that the use of these autonomous procedures or wizard tools by users with limited experience is probably not yet appropriate. Such tools are most appropriately used by users with the experience to accurately judge the quality of the parameter solutions identified.

Future work will involve better numerical methods for combining algorithms into single consistency diagrams and autonomous methods for identifying the best causal (physical) solution are still needed. The application of MAC and related weighted MAC estimates is helpful for detecting contaminated modal vector solutions but numerical solution methods that identify both real-valued modal vectors (normal modes) and complex-valued modal vectors, when appropriate, are still needed. Initial positive results have been obtained, with respect to removing this close mode contamination, by constraining the modal participation factors via real normalization [20]. If this problem can be resolved, this would truly yield an autonomous modal parameter estimation procedure.

\section{Nomenclature}

$N_{i}$ : Number of inputs

$N_{o}$ : Number of outputs

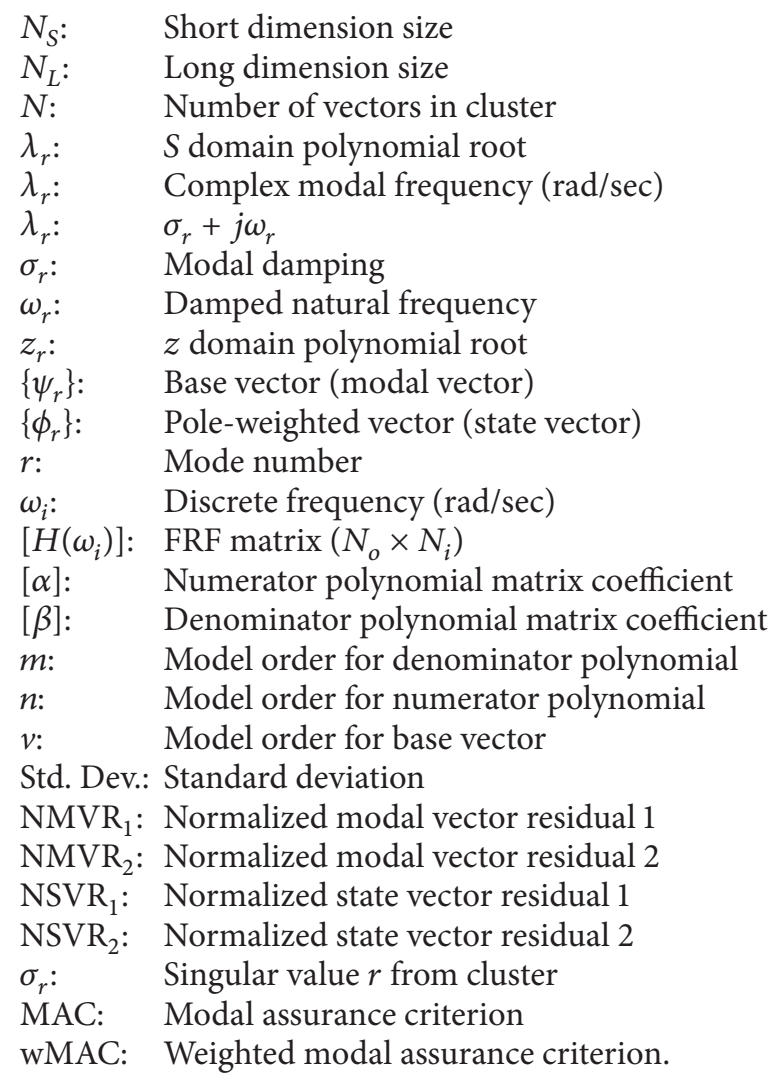

\section{Conflict of Interests}

The authors declare that there is no conflict of interests regarding the publication of this paper.

\section{Acknowledgments}

The authors would like to acknowledge the collaboration and assistance from the graduate students and faculty of the Structural Dynamics Research Lab at the University of Cincinnati. In particular, the discussions and collaborations with Dr. David L. Brown have been instrumental in the progress made to this point.

\section{References}

[1] F. J. Hawkins, "An automatic resonance testing technique for exciting norma 1 modes of vibration of complex structures," in Symposium IUTAM, Progres Recents de la Mecanique des Vibrations Lineaires, pp. 37-41, 1965.

[2] F. J. Hawkins, "GRAMPA—an automatic technique for exciting the principal modes of vibration of complex structures," Royal Aircraft Establishment RAE-TR-67-211, 1967.

[3] G. A. Taylor, D. R. Gaukroger, and C. W. Skingle, "MAMAa aemi-automatic technique for exciting the principal modes of vibration of complex structures," Tech. Rep. ARC-R/M-3590, Aeronautical Research Council, 1967.

[4] R. J. Allemang, D. L. Brown, and W. Fladung, "Modal parameter estimation: a unified matrix polynomial approach," in Proceedings of the 1994 International Modal Analysis Conference, pp. 501-514, 1994. 
[5] R. J. Allemang and D. L. Brown, "A unified matrix polynomial approach to modal identification," in Proceedings of the IndoUS Symposium on Emerging Trends in Vibration and Noise Engineering, pp. 379-390, Indian Institute of Technology, Allied Publishers Limited, New Delhi, India, March 1996.

[6] R. J. Allemang and D. L. Brown, "A unified matrix polynomial approach to modal identification," Journal of Sound and Vibration, vol. 211, no. 3, pp. 301-318, 1998.

[7] R. J. Allemang and A. W. Phillips, "The unified matrix polynomial approach to understanding modal parameter estimation: an update," in Proceedings of the 2004 International Conference on Noise and Vibration Engineering (ISMA '04), pp. 2373-2401, September 2004.

[8] R. J. Allemang, A. W. Phillips, and D. L. Brown, "Combined state order and model order formulations in the unified matrix polynomial method (UMPA)," in Proceedings of the 29th IMAC, a Conference on Structural Dynamics, pp. 429-443, February 2011.

[9] R. J. Allemang, D. L. Brown, and A. W. Phillips, "Survey of modal techniques applicable to autonomous/semi-autonomous parameter identification," in Proceedings of the International Conference on Noise and Vibration Engineering (ISMA '10), p. 42, Katholieke Universiteit Leuven, Leuven, Belgium, 2010.

[10] P. Verboven, B. Cauberghe, E. Parloo, S. Vanlanduit, and P. Guillaume, "User-assisting tools for a fast frequency-domain modal parameter estimation method," Mechanical Systems and Signal Processing, vol. 18, no. 4, pp. 759-780, 2004.

[11] F. Magalhães, Á. Cunha, and E. Caetano, "Online automatic identification of the modal parameters of a long span arch bridge," Mechanical Systems and Signal Processing, vol. 23, no. 2, pp. 316-329, 2009.

[12] F. Magalhães, A. Cunha, and E. Caetano, "Vibration based structural health monitoring of an arch bridge: from automated OMA to damage detection," Mechanical Systems and Signal Processing, vol. 28, pp. 212-228, 2012.

[13] E. Reynders, J. Houbrechts, and G. de Roeck, "Fully automated (operational) modal analysis," Mechanical Systems and Signal Processing, vol. 29, pp. 228-250, 2012.

[14] A. W. Phillips, R. J. Allemang, and D. L. Brown, "Autonomous modal parameter estimation: methodology," in Proceedings of the International Modal Analysis Conference (IMAC '11), 2011.

[15] R. J. Allemang, A. W. Phillips, and D. L. Brown, "Autonomous modal parameter estimation: statisical considerations," in Proceedings of the 29th IMAC, a Conference on Structural Dynamics (IMAC '11), pp. 385-401, February 2011.

[16] D. L. Brown, R. J. Allemang, and A. W. Phillips, "Autonomous modal parameter estimation: application examples," in Modal Analysis Topics, vol. 3 of Conference Proceedings of the Society for Experimental Mechanics Series, pp. 403-428, 2011.

[17] R. J. Allemang and D. L. Brown, "A correlation coefficient for modal vector analysis," in Proceedings of the International Modal Analysis Conference, pp. 110-116, 1982.

[18] R. J. Allemang, “The modal assurance criterion (MAC): twenty years of use and abuse," Sound and Vibration Magazine, vol. 37, no. 8, pp. 14-23, 2003.

[19] F. Deblauwe and R. J. Allemang, "A possible origin of complex modal vectors," in Proceedings of the 11th International Seminar on Modal Analysis, p. 10, Katholieke Universiteit Leuven, Leuven, Belgium, 1986.
[20] A. W. Phillips and R. J. Allemang, "Normalization of experimental modal vectors to remove modal vector contamination," in Proceedings of International Modal Analysis Conference (IMAC '14), 2014. 

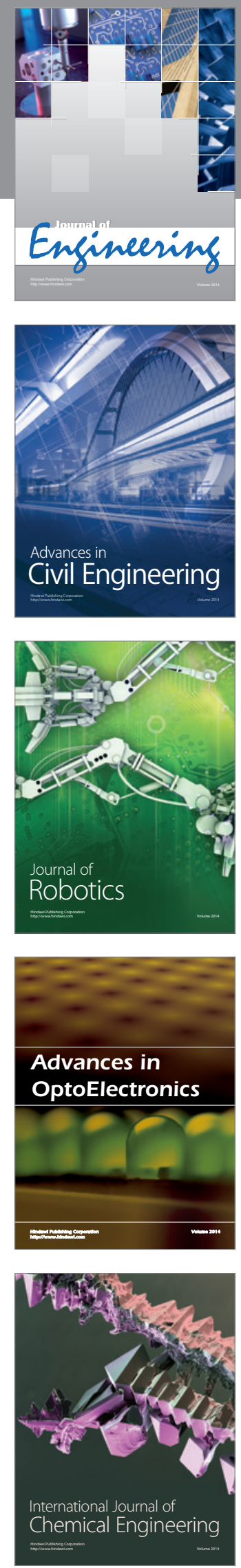

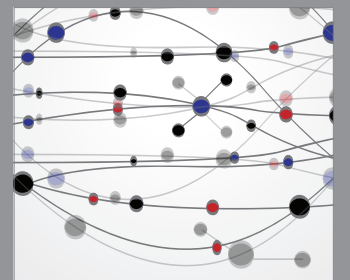

The Scientific World Journal
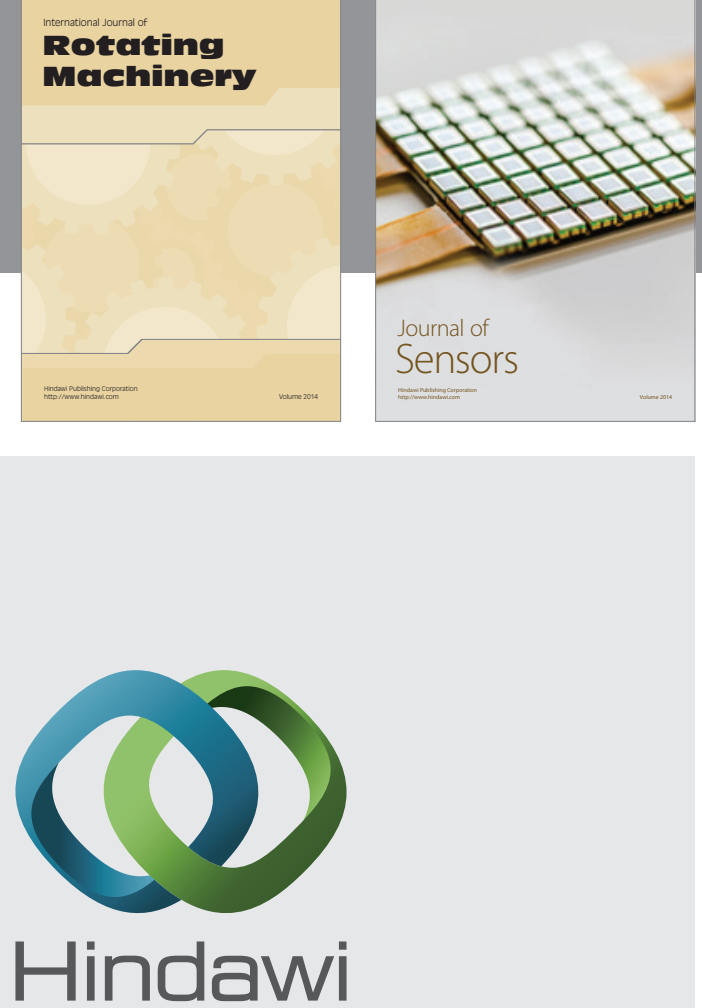

Submit your manuscripts at http://www.hindawi.com
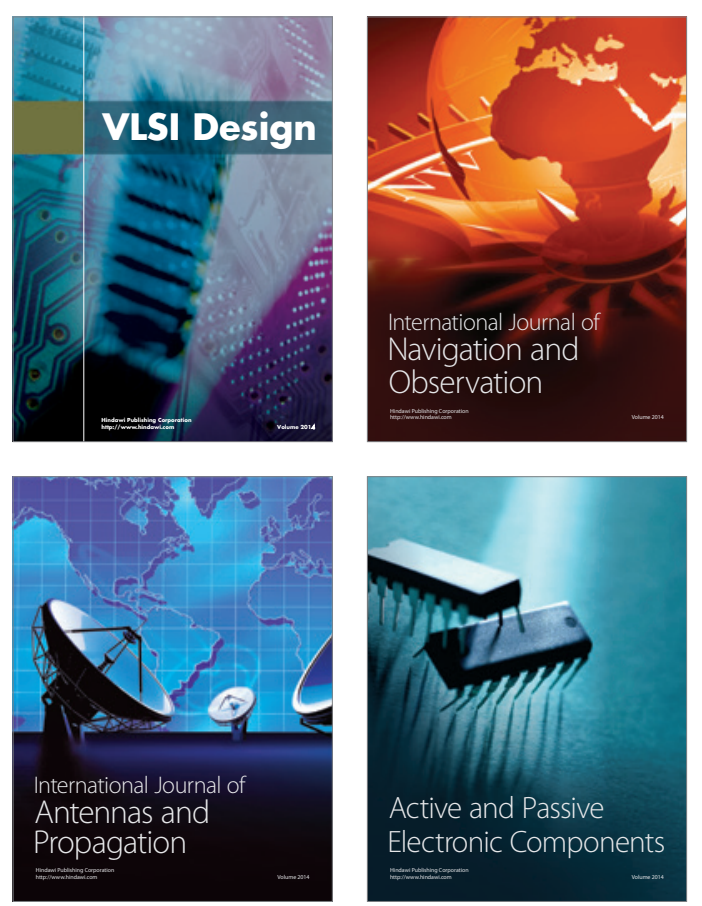
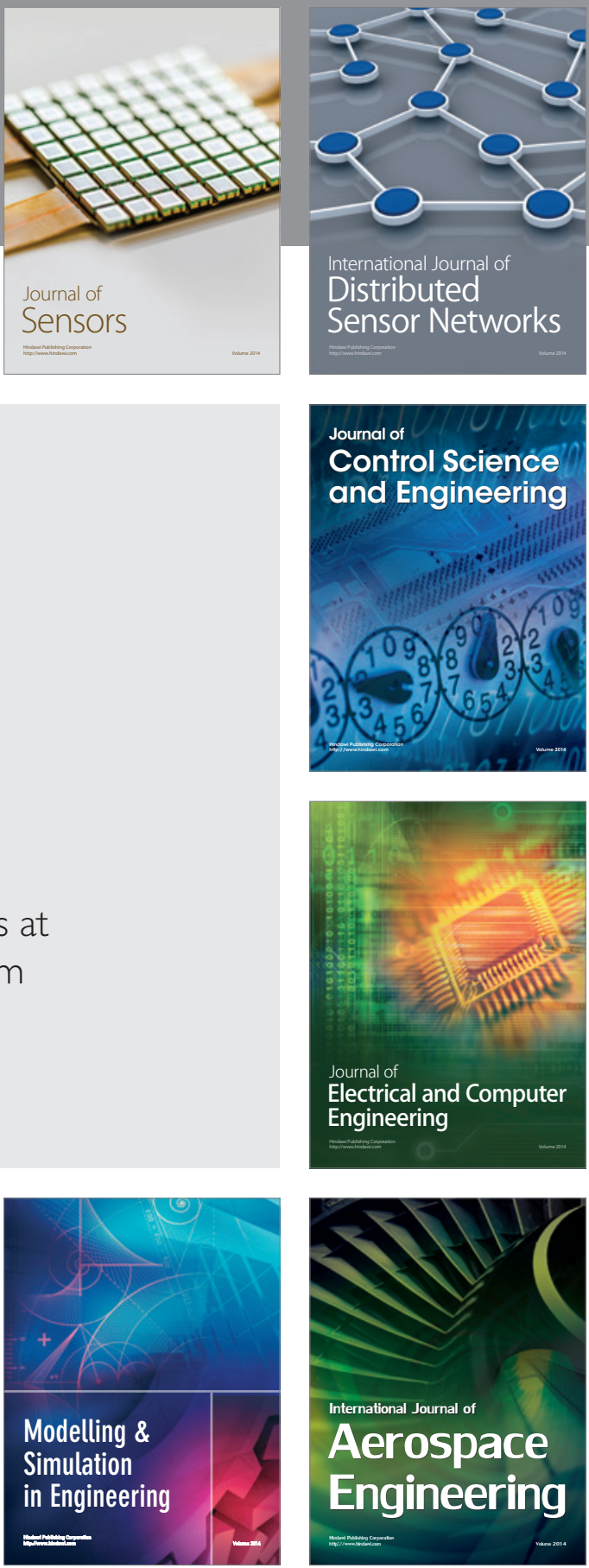

Journal of

Control Science

and Engineering
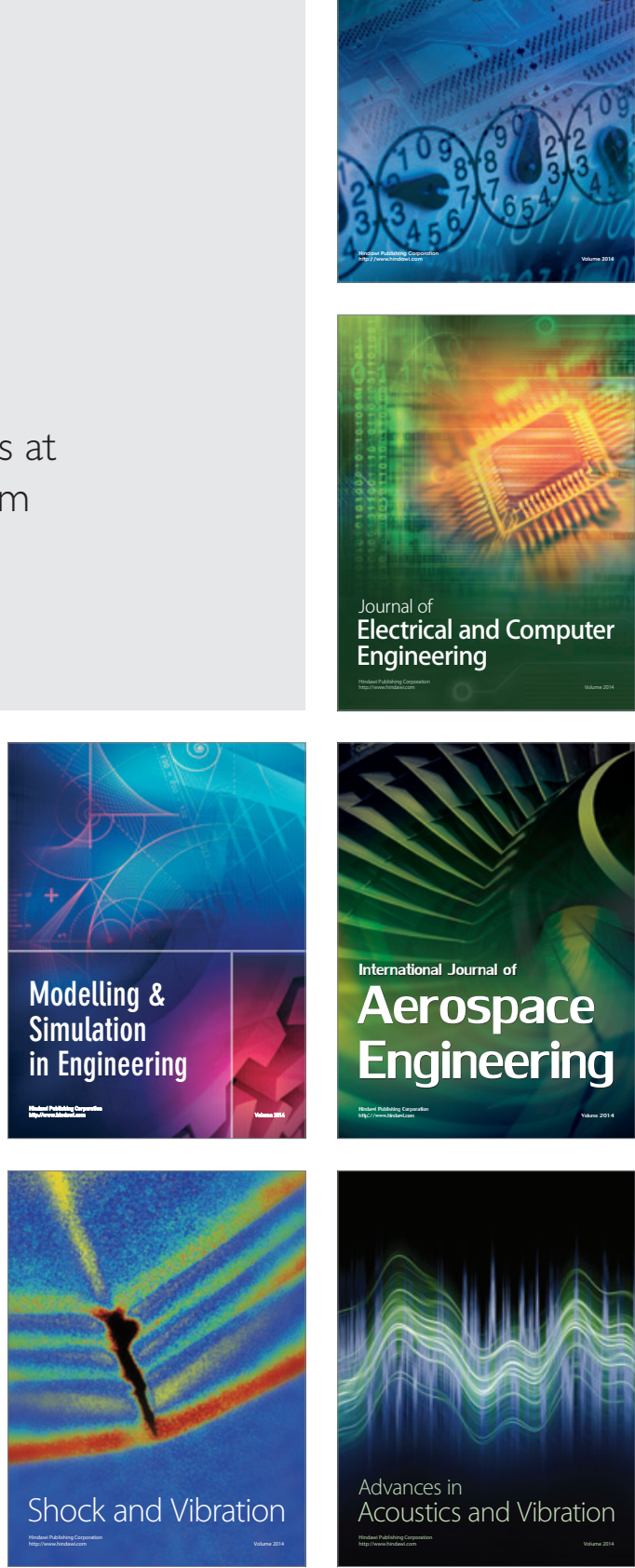